\title{
Theoretical Study on Influence Factors of the Angle of Inner Hole Generatrix through Cone Hole Open-die Cold Extrusion
}

\author{
Lei MA ${ }^{1, a}$, Peng-Biao HAN ${ }^{2, b, *}$, Xiao-Lei $J^{3, c}$, Yu-Xi CHEN ${ }^{4}$, Jian-Ying CUI ${ }^{5}$, \\ Su-Ling $\mathrm{LU}^{6}$, Jun $\mathrm{LI}^{7}$ \\ ${ }^{1,2,3,6}$ College of Material Science and Engineering of Hebei University of Science and Technology, \\ Shijiazhuang, China \\ 4,5Juli Sling Company Limited, Baoding, China \\ ${ }^{7}$ Hebei GEO University, Shijiazhuang, China \\ a416412410@qq.com, bhpb68@163.com, ${ }^{c} 1203052021 @ q q . c o m$ \\ ${ }^{*}$ Corresponding author
}

Keywords: Cone Hole, Open-die Cold Extrusion, Upper-bound Method, Stream Function, Unstable Velocity Field.

\begin{abstract}
The plastic deformation characteristic of cone hole open-die cold extrusion was analyzed based on the concepts of hydrodynamics, the stream function of instantaneous velocity field was built. According to the determination of the equation of stream function, the kinematically admissible continuity unstable velocity field and the shape and position of its relevant velocity discontinuity were built. The factors of velocity field were analyzed. The theoretical model of extrusion power was obtained by the upper bounding theory. The factors of the angle of the cone hole generatrix were analyzed by programming and calculating.
\end{abstract}

\section{Introduction}

Hole machining plays an important role in machining operation. Because of its low production efficiency, high energy consumption, difficulty in processing. Especially, its difficulty in machining inner cone hole. the technology of open-die cold extrusion was needed, and it's been applied to the machining of deep tine cone hole, special-shaped hole, tapered pipe, curved generatrix holes and other parts. The cone hole open-die cold extrusion forming process was studied based on the forming technology, the main influencing factors of the cone hole was determined. It is significant to forming technology of the cone hole formed by using open-die cold extrusion.

\section{The Establishment of Upper Bound Model}

The Concept of Upper Bound Stream Function Method in the Application of Open-die Cold Extrusion

Streamline and streamline equation: at a certain instant, the velocity vector direction of each point of the speed field is connected together to form a curve, which is called streamline. According to the definition of streamline, in the fluid mechanics the streamline differential equation of the rectangular coordinate system as follow ${ }^{[2,3]}$ :

$$
\frac{v_{x}(x, y, z, t)}{d x}=\frac{v_{y}(x, y, z, t)}{d y}=\frac{v_{z}(x, y, z, t)}{d z}
$$

The streamline equation of axisymmetric open-die cold extrusion as follow:

$$
\frac{v_{r}(r, z, t)}{d r}=\frac{v_{z}(r, z, t)}{d z}
$$


The stream function is defined according to the continuity equation, that is, satisfying the continuous equation is the existence condition of the stream function. According to the continuity equation in cylindrical coordinates, the stream function is as follow:

$$
d \psi=-r v_{z} d r+r v_{r} d z=\frac{\partial \psi}{\partial r} d r+\frac{\partial \psi}{\partial z} d z
$$

The relationship between stream function and velocity field as follow [2]:

$$
v_{r}=-\frac{1}{r} \frac{\partial \psi}{\partial z} ; v_{z}=\frac{1}{r} \frac{\partial \psi}{\partial r}
$$

\section{Velocity Field}

(I) Area is a rigid area, the velocity field can be directly determined. (П) Area is the deformation area. (П) Area velocity field is obtained according to the stream function and the relationship of equation stream function equation and stream function and velocity field. The overall velocity field of the extrusion process is as follow:

$$
\left\{\begin{array}{l}
v_{r}=0, v_{z}=v_{0} \\
v_{r}=-\frac{v_{0}\left[R_{0}^{2}(t)-r_{0}^{2}\right]}{r\left[R_{d}^{2}(z)-R_{m}^{2}(z)\right]^{2}}\left\{R_{d}(z) \tan \alpha\left[R_{m}^{2}(z)-r^{2}\right]+R_{m}(z) \frac{r_{0}-r_{1}(t)}{\mathrm{h} 1(t)}\left[r^{2}-R_{d}^{2}(z)\right]\right\} \\
v_{z}=\frac{v_{0}\left[R_{0}^{2}(t)-r_{0}^{2}\right]}{\left[R_{d}^{2}(z)-R_{m}^{2}(z)\right]}
\end{array}\right.
$$

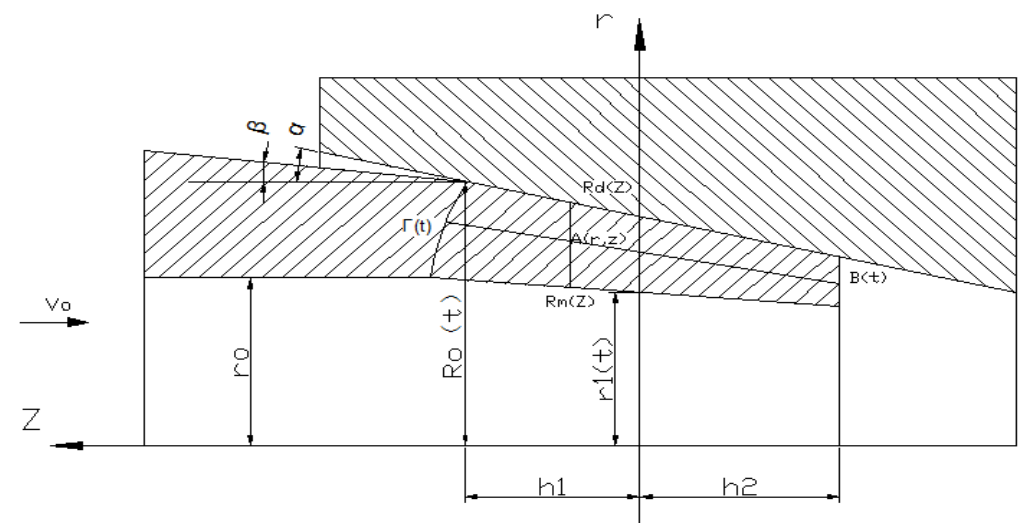

Fig.1 Parameter Sketch of the Cone Hole Open-die Cold Extrusion

\section{Selection of Plastic Deformation Area and Velocity Discontinuity Line}

Figure 1 shows that the plastic deformation area is composed of $\Gamma(t), B(t), R_{d}(z)$ and $R_{m}(z)$.

(1) According to the definition of streamline, $R_{m}(z)$ should be a streamline, the flow of the flow tube is zero. Stream function $\Psi=0[2]$. The equation of $R_{m}(z)$ as follow:

$$
\begin{aligned}
& R_{m}(z)=r_{1}(t)+z \frac{r_{0}-r_{1}(t)}{\mathrm{h} 1(t)} \\
& R_{m}^{\prime}(z)=\frac{r_{0}-r_{1}(t)}{\mathrm{h} 1(t)}
\end{aligned}
$$


(2)Analysis of the location of $\Gamma(t)$. The premise of the open cold extrusion technology can be achieved is that the billet should keep a good rigidity before the contact with the mold $[2,5]$. In order to determine the location and time of $\Gamma(t)$, suppose rigid region (I) always make rigid translation in the main deformation stage. According to the geometric relationship, the position function of $\Gamma(t)$ and the equation of $R_{0}(t)$ as follow:

$$
\begin{aligned}
& \mathrm{h} 1(t)=\frac{\sin \beta \cos \alpha\left|v_{0} t\right|}{\sin (\alpha-\beta)} \\
& R_{0}(t)=R_{1}+\frac{\sin \beta \sin \alpha\left|v_{0} t\right|}{\sin (\alpha-\beta)}
\end{aligned}
$$

(3)Analysis of the location of $B(t) . B(t)$ is the small end boundary line of the product, and it will have small deformation in the process of shrinking. Its deformation can be ignored, so it can be treated as a straight line.

\section{Calculation of Plastic Deformation Power}

\section{Plastic Deformation Power}

Deformation area velocity field as follow:

$$
\left\{\begin{array}{l}
\dot{\varepsilon}_{r}=\frac{-v_{0}\left[R_{0}(t)^{2}-r_{0}^{2}\right]}{\left[R_{d}(z)^{2}-R_{m}(z)^{2}\right]^{2}}\left\{R_{m}(z) \frac{r_{0}-r_{1}(t)}{\mathrm{h}_{1}(t)}\left[1+\frac{R_{d}(z)^{2}}{r^{2}}\right]+R_{d}(z) \tan \alpha\left[1+\frac{R_{m}(z)^{2}}{r^{2}}\right]\right. \\
\dot{\varepsilon}_{\theta}=\frac{-v_{0}\left[R_{0}(t)^{2}-r_{0}^{2}\right]}{\left[R_{d}(z)^{2}-R_{m}(z)^{2}\right]^{2}}\left\{R_{d}(z) \tan \alpha\left[\frac{R_{m}(z)^{2}}{r^{2}}-1\right]+R_{m}(z) \frac{r_{0}-r_{1}(t)}{h_{1}(t)}\left[1-\frac{R_{d}(z)^{2}}{r^{2}}\right]\right. \\
\dot{\varepsilon}_{\mathrm{z}}=\frac{-2 v_{0}\left[R_{0}(t)^{2}-r_{0}^{2}\right]}{\left[R_{d}(z)^{2}-R_{m}(z)^{2}\right]^{2}}\left[R_{d}(z) \tan \alpha-R_{m}(z) \frac{r_{0}-r_{1}(t)}{\mathrm{h}_{1}(t)}\right] \\
\dot{\gamma}_{r z}=\frac{-v_{0}\left[R_{0}(t)^{2}-r_{0}^{2}\right]}{2\left[R_{d}(z)^{2}-R_{m}(z)^{2}\right]^{3}}\left\{\left[r^{2}-R_{d}(z)^{2}\right]\left[\frac{r_{0}-r_{1}(t)}{\mathrm{h}_{1}(t)}\right]^{2} \times\left[3 R_{m}(z)^{2}+R_{d}(z)^{2}\right]+4 R_{d}(z) R_{m}(z) \tan \alpha \frac{r_{0}-r_{1}(t)}{\mathrm{h}_{1}(t)} \times\right. \\
\left.\quad\left[R_{m}(z)^{2}-2 r^{2}+R_{d}(z)^{2}\right]-\left[R_{d}(z)^{2}-r^{2}\right] \tan ^{2} \alpha \times\left[3 R_{d}(z)^{2}+R_{m}(z)^{2}\right]\right\}
\end{array}\right.
$$

The equation of plastic deformation power as follow:

$$
\dot{W}_{i}=\sqrt{2} K \int_{V} \sqrt{\dot{\varepsilon}_{i j} \dot{\varepsilon}_{i j}} d V
$$

\section{Intermittent Power}

The equation of velocity discontinuity as follow:

$$
\dot{V}_{\Gamma}=v_{r / z=h_{1}(t)}=\frac{v_{0}}{r\left[R_{0}(t)^{2}-r_{0}^{2}\right]}\left[R_{0}(t) \tan \alpha\left(r_{0}^{2}-r^{2}\right)+r_{0} \frac{r_{0}-r_{1}(t)}{h_{1}(t)}\left[r^{2}-R_{0}(t)^{2}\right]\right.
$$

The equation of intermittent power as follow:

$$
\dot{W}_{d}=\frac{2 \pi \sigma_{s}}{\sqrt{3}} \int_{r_{0}}^{R_{0}(t)}\left|\dot{V}_{\Gamma}\right| r d r
$$

\section{Friction Power}

The equation of velocity discontinuity as follow: 


$$
v_{f}=v_{z / r=R_{d}(z)} \cos \alpha+v_{r / r=R_{d}(z)} \sin \alpha=\frac{v_{0}\left[R_{0}(t)^{2}-r_{0}^{2}\right]}{R_{d}(z)\left[R_{d}(z)^{2}-R_{m}(z)^{2}\right]} \sin \alpha+\frac{v_{0}\left[R_{0}(t)^{2}-r_{0}^{2}\right]}{\left[R_{d}(z)^{2}-R_{m}(z)^{2}\right]} \cos \alpha
$$

The equation of friction power as follow:

$$
\dot{W}_{f}=m \cdot \frac{2 \pi \sigma_{s}}{\sqrt{3}} \int_{H_{2}(t)}^{H_{1}(t)}\left|v_{f}\right| R_{d}(z) / \cos \alpha d z
$$

\section{Total Extrusion Power}

Total extrusion power as follow:

$$
\dot{W}_{\alpha}^{k}=\dot{W}_{d}+\dot{W}_{\mathrm{i}}+\dot{W}_{f}
$$

In the design of the mold deformation, if the size of the blank is known, and only one coordinate that bore radius $\mathrm{r} 1 \mathrm{t}$ ) coordinate system on the vertical axis is not determined in the integral function and the integral limit of power of each deformation. According to the upper bound method, the R1 (t) can be determined by optimizing the upper bound power, thereby the generatrix cone hole can be determined. Then the length of the part after the extrusion is obtained according to the condition of constant volume.

The numerical integration, power calculation and process parameter calculation are carried out using the method of Gauss five points double integral and Gauss five point one integral. The block diagram of program and is omitted.

\section{Results and Discussion}

\section{Analysis for Factors of the Cone Hole Generatrix Angle}

The main factors of the change of the cone hole Generatrix angle after extrusion are the original thickness to diameter ratio of the blank, blank external cone angle and small end diameter etc.

The influence of these factors on the cone hole Generatrix angle are analyzed and calculated based on theoretical model, the results are as follows.

\section{The Influence of the Ratio of the Thickness to Diameter of the Blank}

The definition of the ratio of the thickness to diameter of the blank is:

$\mathrm{t} 0 / \mathrm{D} 0=(\mathrm{D} 0-\mathrm{d} 0) / 2 \mathrm{D} 0$

D0--the small end diameter of blank

d0--diameter of inner hole of blank

The influence of the ratio of the thickness to diameter of the blank is shown in Fig.2. Fig.2 shows that in the case of other parameter conditions do not change, the relative cone angle $\gamma / \beta$ increases linearly with the increase of the ratio of the thickness and diameter of the blank t0/D0 in a certain range.

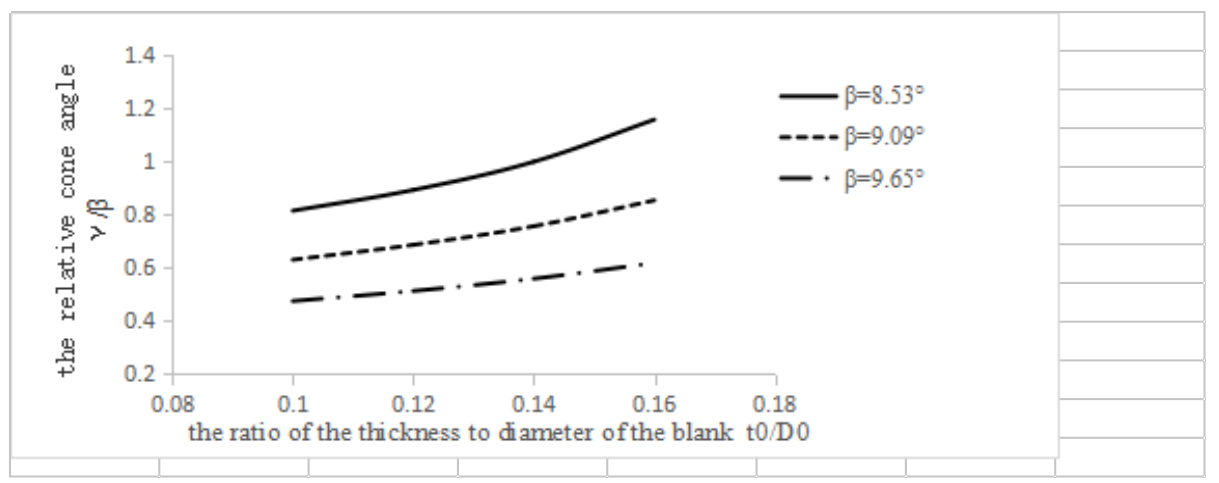

Fig.2 The Influence of the Ratio of the Thickness to Diameter of the Blank to Relative Cone Angle 


\section{The Influence of the Blank External Cone Angle}

The influence of the blank external cone angle on the cone hole of the part is shown in Fig.3. Figure 3 shows that, the relative cone angle $\gamma / \beta$ substantially decreases linearly with the blank external cone increases.

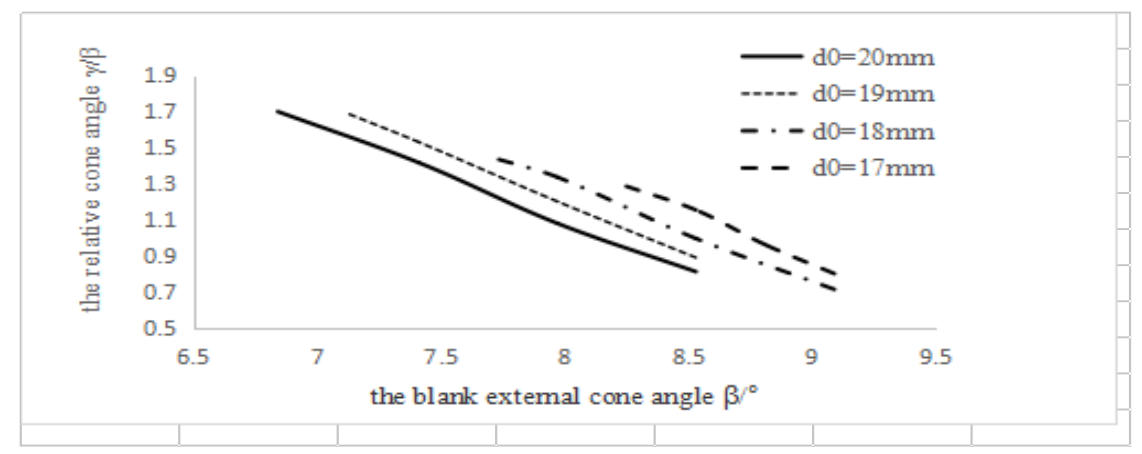

Fig.3 The Influence of the Blank External Cone Angle to Relative Cone Angle

\section{Other Factors}

According to the theoretical power formula, the cone hole of the part is not only related to the above factors, but also to the mold cone angle, lubrication conditions and material properties etc. The lubrication conditions and material properties both have certain influence on the forming process of the power, but have no effect on the forming of cone hole.

\section{Conclusion}

(1) Stream function was introduced, and the dynamic soluble unsteady state continuous velocity field of the open-die cold extrusion of the cone hole was established.

(2) Based on the velocity field model established in this paper, the theoretical calculation was carried out by using the upper bound method. The influence of the main factors on the cone hole was obtained: the ratio of inner hole taper to outer taper of blank (the relative cone angle) increases linearly with the increase of the ratio of the thickness to diameter of the blank within a certain range; the relative cone angle decreases with the blank outer cone angle increases.

\section{References}

[1] LI Jun. Research on open-die cold extrusion forming's modelling and its technological parameters application [D]. Tianjin: Tianjin University, 1997.

[2] Lu Suling. Study on the prodiction of size and shape for open-die extrusion of deep thin cone-hole workpiece [D]. Qinghuangdao: Master Thesis of Yanshan University.

[3] Li Jun, Han Pengbiao, Zhang Shuangjie. Research on velocity field modelling of open-die cold extrusion by stream function [J]. Forging and Stamping Technology, 2000, 03:3-5.

[4] HAN Pengbiao, Lu Suling, LI Jun, Zhang Shuangjie. Theoretics study on the production of size and shape for open-die extrusion of deep thin cone-hole workpiece [J]. Journal of Plasticity Engineering, 2008, 04:105-107+125.

[5] LI Jun, HAN Pengbiao. Theoretical and experimental study on maximum reduction of open-die cold extrusion[J]. Journal of Plasticity Engineering, 2000, 2(7):31-34. 